\title{
Modelling of Shapes without Landmarks
}

\author{
Felix Wehrmann and Ewert Bengtsson \\ Uppsala University, 75237 Uppsala, Sweden, \\ felix@cb.uu.se, \\ http://www.cb.uu.se/ ${ }^{\text {felix }}$
}

\begin{abstract}
The complexity in variation that objects are provided with motivates to consider learning strategies when modeling their shape. This paper evaluates auto-associative neural networks and their application to shape analysis. Previously, such networks have been considered in connection with 'point distribution models' for describing two-dimensional contours in a statistical manner. This paper suggests an extension of this idea to achieve a more flexible model that is independent of landmarks.
\end{abstract}

\section{Introduction}

It is a challenging problem to formulate models that represent the shape of objects. Questions about global or local features naturally appear when it comes to descriptions of their characteristics. If data is available about the desired class of objects, learning strategies can be used to extract the essence of shape from given examples. In this paper, a model is presented that learns the variation of shape within its class by establishing its individual feature space.

Obviously, a model that is based on general pre-assumptions, e.g. to adapt to any class of shape that is found in nature, needs to consider the entire data about the shapes in question, so that accidental loss of essential information is prevented. In particular, systematic processing, like e.g. filtering, eventually yields unwanted effects.

Several approaches have been carried out to cover the variety in the appearance of shapes. Initially focused on two-dimensional shapes, there has been mainly two concepts of modelling.

In 1992, Staib et al. introduced Fourier descriptors to contours in [7]. Fourier methods derive frequency information about the points of a contour, collecting information about the frequency behaviour of a shape. Restrictions on the number of harmonics in the decomposition control the degree of generality and enable a low dimensional description. However, essential spatial information can get lost by the decomposition.

A more recent approach by Cootes et al. [2] introduces 'point distribution models' (PDM) in 1995. With the same purpose as above, dimensionality is reduced to obtain a compact description of shape. In PDMs, each sample of a training set is considered a point in a hyper-space. The model is derived from a principle component analysis (PCA) of the covariances within the training set. 
It therefore requires point-to-point correspondences between the contours, i.e. landmarks.

A drawback of PDM is the assumption that the landmarks in the shape data form a ellipsoid-like distribution in the shape space, thus satisfying linear models. However, shapes often provide non-linearities, e.g. under rotation. Therefore, if possible, training shapes should be carefully aligned prior to training of the PDM.

In [6], non-linearity is addressed and an alternative model is presented. Considering non-linear modelling, the landmarks are allowed to vary on curved paths within the population. Here, a neural network performs a non-linear PCA that makes it possible to describe distributions that are far from being ellipsoids.

A central issue about PDM is that it is necessary to depict landmarks on a contour. Interestingly, the landmarks, which serve as input to the training procedure of the model, form a recognition problem involving models itself. Therefore, for reasons of practicality, landmarks are often extracted manually, which is time consuming. Under the scope that we want to collect knowledge about the variations within a class, the composition of landmarks will always prohibit an analysis which goes beyond the quality of that set.

Our aim is to provide a more general model that is independent of landmarks. It turns out that a solution would involve non-linear functions to describe the allowed variations of a contour. In this paper, we apply a similar network as used in [6] to $2 \mathrm{D}$ contours of shape. This type of network is, as discussed in the next section, well suited for the presented task as it can be considered a non-linear PCA with adjustable kernel functions. In contrast to [6], we show that non-linear modelling allows for modelling with the landmarks being omitted.

This paper restricts itself to a discussion on modelling, and does not consider detection of shapes in images. Therefore, training data is generated artificially, providing only desired variations. Then, an auto-associative neural network (AANN) is composed from two non-linear networks and trained on the data. It will create an implicit representation of the data. Finally, the trained network is separated again, providing one network for encoding and one for reconstruction of shape.

\section{Methods}

\subsection{Shape data}

The shape data we are about to generate is used to train and verify the model. In our experiments, we assume that the measurements about shape represent the location of a contour along the boundary of an object. For simplicity, we consider objects in two dimensions, however, this is no general restriction of the procedure. The contour is measured at $N^{\prime}$ regularly distributed and ordered points $p$, thus yielding a set of $N=2 \times N^{\prime}$ coordinates $x_{i}$. This set is aligned in a vector $\boldsymbol{x}$ of $N$ elements, called shape vector. Therefore, it is also considered a single point in an $N$ dimensional shape space, where a smooth variation on the original shape would result in a continuous displacement of the point location. 

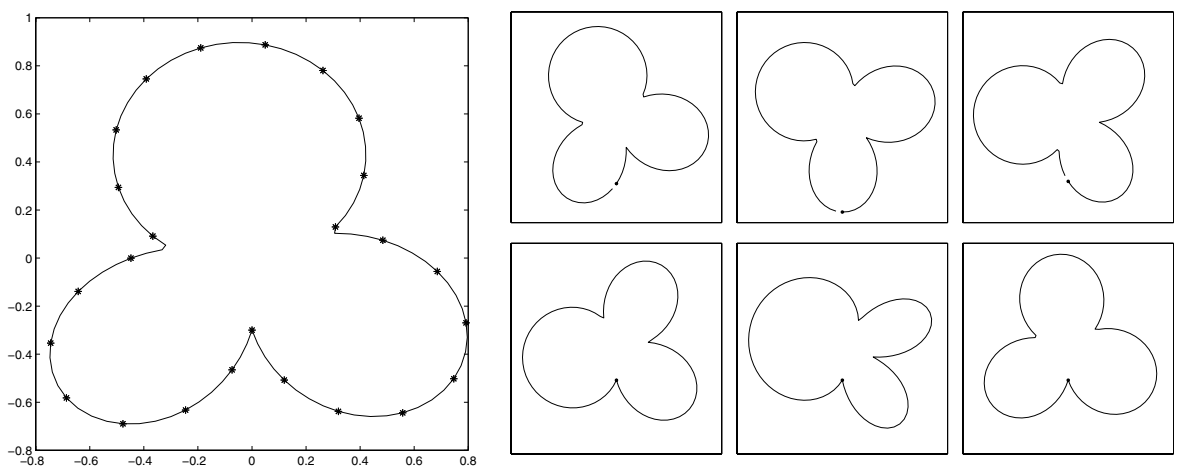

Fig. 1. Shape function with regularly spaced sample locations marked with stars (left). Shapes from the rotation training set at 30,60, and 90 degrees. The beginning of the sampling is marked with a star (upper row). Examples from the deformed training set (lower row).

The shape data used here is artificial. It is therefore possible to explicitly generate training data with a desired type of variation for investigation purposes.

How do we arrive at landmark-free shape vectors? An "artificial retina" is a sensor phalanx where the location of each sensor is fixed in relation to its neighbours. We will assume that the measurements $x_{i}$ are provided by an artificial retina which can move freely along the shape to be measured. As a consequence, a variation in the shape of an object will not make a point $p$ follow a certain landmark related to the shape, but instead slide along the contour. Points therefore cover different areas on the shape independently of landmarks.

A class of shapes is defined by a continuous representation of a parameterized contour $f(t)$ (see Fig. 1, left) including a number of transformations that reflect variation. The mapping function writes

$$
f: R^{M} \rightarrow R^{N}, \boldsymbol{\theta} \rightarrow f(t, \boldsymbol{\theta}), t=0 . .1
$$

where $\boldsymbol{\theta}$ controls the (stochastic) transformations.

To produce a single sample $\boldsymbol{x}$ from the class, the desired $\boldsymbol{\theta}$ is chosen and the transformed contour is sampled according to the specifications of the artificial retina, i.e. at $N^{\prime}$ points of equal distance, and the co-ordinates are stored in $\boldsymbol{x}$.

Two kinds of transformations are considered, as depicted in Fig. 1, right. A global transformation that provides explicit non-linear support is simple rotation. The non-linearity is apparent by the non-linearity of the contour itself, since there are no landmarks that would follow the rotation of the object from the viewpoint of the artificial retina. Note, that translation or scaling would not provide the desired variations, because they are linear transformations.

A local transformation is realised as a stochastic deformation, i.e. the shape deflects locally from the initial contour according to a stochastic factor, which is drawn from a normal distribution. 


\subsection{Neural network model}

The heart of the model is a non-linear AANN, as presented in Fig. 2. This particular type of network has originally been used for data compression of different kinds, and is used here to learn the mapping $f$. The particular design of the network makes it suitable for modelling the desired variations in the data for two reasons. First, the network is capable of representing highly non-linear functions, a feature which lies partly in the transfer function and partly in the inter-connection scheme of the neurons.

Second, the network establishes an intermediate representation of the data in a so called feature space. Since no information about the initial mapping $f$ is available, we would not have anything to train the network on. Here, the trick is to associate the feature space of the trained network with the parameter space of $\boldsymbol{\theta}$. The feature space is spanned by the neurons of the smallest layer, because here we can assume the most compact representation of the training data.

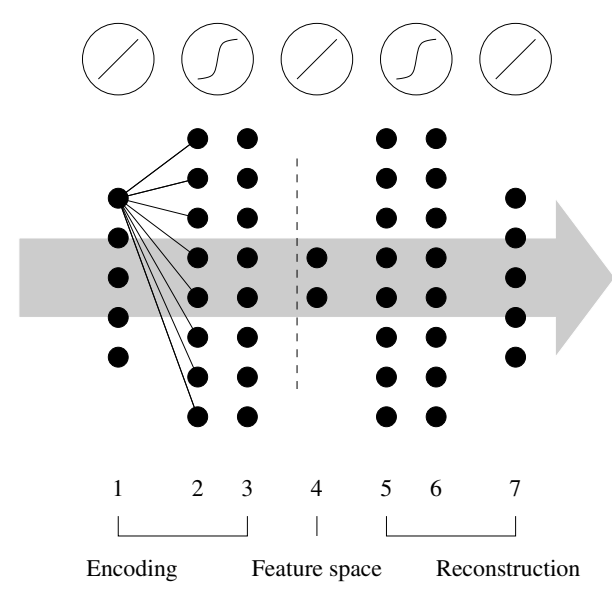

Fig. 2. An auto-associative neural network is composed of two feed-forward networks (glued together at the dotted line). After training, the left network performs a mapping from shape space into feature space, while the right one performs the inverse mapping used to reconstruct shape vectors. Layers $2,3,5$, and 6 have sigmoidal transfer functions to model non-linearities, layers 1,4 , and 7 are linear nodes, as common for a function approximator. The network is fully interconnected as sketched in layers 1, 2 for a single neuron.

Auto-associative networks are trained supervised, where the input and output data are pairwise the same. The model is a feed-forward neural network and thus can be trained using common backpropagation strategies. We tested different gradient based methods (gradient decent with/without momentum), as well as resilient backpropagation (RProp). The latter yielded fastest convergence and turned out to be stable. 

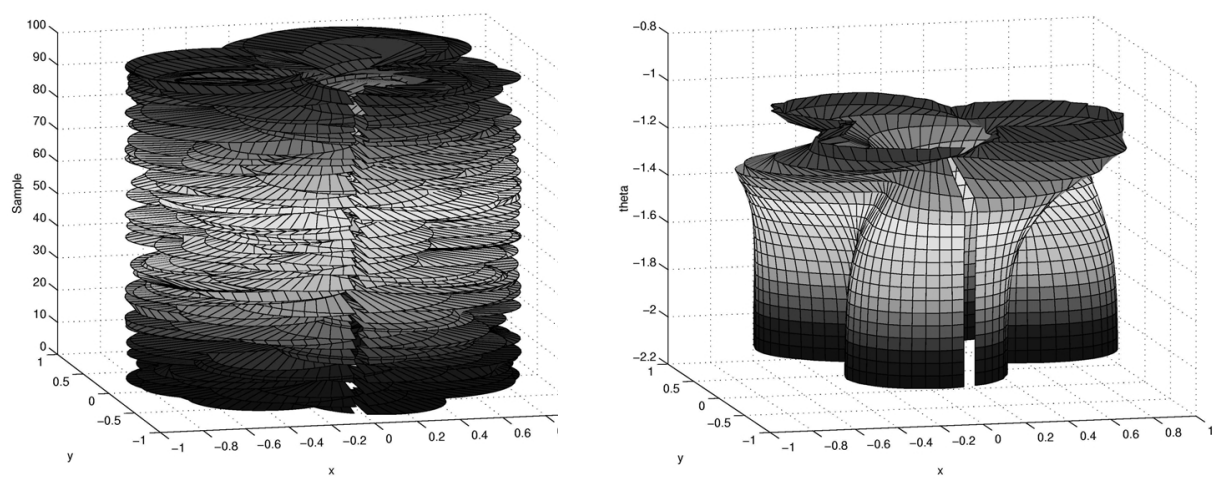

Fig. 3. A stack of 100 rotated shapes used to train the AANN (left). Simulation of the reconstruction part of the network for the score interval (right). Parameter $\boldsymbol{\theta}$ varies along the vertical axis. Interestingly, the network only represents 120 degrees of rotation due to symmetries in the shape itself.

\section{Results}

The AANN is trained on shape vectors until convergence of the residual error. After training, the network configuration is not changed anymore.

Simulating only the left part of the AANN, having shape vectors forming the input of the network, corresponds to a non-linear projection of the shape data into the feature space. The obtained scores, that is data configurations in the feature space, compare to the corresponding parameter $\boldsymbol{\theta}$. In particular, the appearance of shapes is characterised by the distribution of the scores. It is, however, not possible to force the AANN to establish a feature space that is in exact correspondence to $\boldsymbol{\theta}$. The network approaches the score distribution iteratively from randomized initial weights. Therefore, a by-value comparison is usually biased by offsets and scalings which stem from the initialization. Consequently, two networks having different initializations usually establish different feature spaces when trained on the same data.

Simulating the right part of the network on scores drawn from the distribution in the feature space, shape vectors are reconstructed for verification.

The experiments are carried out on two training sets covering rotation and deformation. Shapes from each class were sampled at 100 regularly spaced points, yielding shape vectors in $N=200$ dimensions.

The AANN has $N$ inputs and $N$ outputs, and creates a feature space of one single parameter $M=1$. Note that the value of $M$ matches the dimensionality of $\boldsymbol{\theta}$ in the training set. In the rotation training set $\boldsymbol{\theta}$ naturally compares to the rotation angle. In both the encoding part and the reconstruction part, two non-linear hidden layers are active, containing 10 nodes each.

The rotation training set consists of 100 samples (see Fig. 3). The network is trained on the set for 1000 epochs, which yielded convergence. Each input 

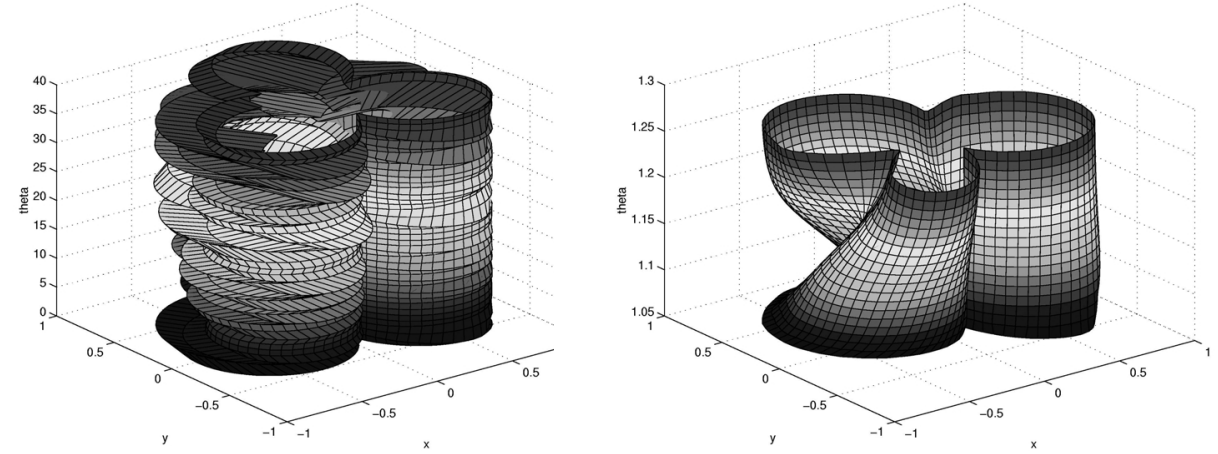

Fig. 4. 40 deformed training shapes stacked together arbitrarily (left). Reconstructed shapes corresponding to the score interval obtained by simulating the trained network (right). Varying $\boldsymbol{\theta}$, plotted along the vertical axis, provides a sweep over the learned variations.

produced a score in the feature space, corresponding to the encoding of a shape. For the whole set, the interval for the scores was within $-2.2<\boldsymbol{\theta}<-1$.

The deformation training set comprises 40 samples, which are shown in Fig. 4. Again, 1000 epochs yielded convergence under training. Scores for this training set are found within $1.07<\boldsymbol{\theta}<1.26$.

\section{Conclusion}

We introduced a trainable model that learns variations of shape from examples by means of AANN. The model establishes a low dimensional representation of the characteristics of shape in a feature space. It is an important quality of the model to capture non-linear relations between the measurements. Therefore, the set of allowed classes for this type of model can be extended by landmark-free contours, as we were able to show addressing the introduced classes of shape above.

However, it is important to note that the AANN learned rotation and deformation only according to a particular class. It is therefore not in the position to learn a generally applicable rule of object rotation, because rotation is considered only a, however complex, variation within a class. Accordingly, it would generally be impossible for the trained network to detect rotation of shapes from unknown classes.

From the discussions it turned out that in the absence of landmarks, the nonlinearity of the curvature of a contour itself demands for non-linear models due to sampling according to an artificial retina. This is in contrast to the landmark case, where relationships still can be linear.

Learning variation of shapes from examples in a more general way can improve modelling and recognition tasks as they often are required in imaging 
applications. Besides stronger, more accurate models, the generation of training data is simplified because landmark detection can be avoided.

\section{References}

1. David P. Casasent and Leonard M. Neiberg. Classifier and shift-invariant automatic target recognition neural networks. Neural networks, 8(7-8):1117-1129, 1995.

2. T. F. Cootes, C. J. Taylor, D. H. Cooper, and J. Graham. Active shape modelstheir training and application. Computer vision and image understanding, 61(1):3859, 1995.

3. Daniel Freedman. Efficient simplicial reconstructions of manifolds from their samples. IEEE transactions on pattern analysis and machine intelligence, 24(10):13491357, October 2002.

4. Michael A. Sipe, David P. Casasent, and Leonard M. Neiberg. Feature space trajectory representation for active vision. SPIE, 1997.

5. P.D. Sozou, T.F. Cootes, C.J. Taylor, and E.C. Di Mauro. Non-linear generalization of point distribution models using a multi-layer perceptron. Image and Vision Computing, 13(5):451-457, June 1995.

6. P.D. Sozou, T.F. Cootes, C.J. Taylor, and E.C. Di Mauro. Non-linear point distribution modelling using a multi-layer perceptron. Image and Vision Computing, 15(6):457-463, 1997.

7. Lawrence H. Staib and James S. Duncan. Boundary finding with parametrically deformable models. IEEE transactions on pattern analysis and machine intelligence, 14(11):1061-1075, November 1992. 